\title{
Modal analysis of a small surface emitting laser with a selectively oxidized waveguide
}

\author{
K. L. Lear, ${ }^{\text {a) }}$ K. D. Choquette, R. P. Schneider, Jr., and S. P. Kilcoyne \\ Sandia National Laboratories, Photonics Research Department 1312, MS 0603, P. O. Box 5800, \\ Albuquerque, New Mexico 87185-0603
}

(Received 6 February 1995; accepted for publication 16 March 1995)

\begin{abstract}
We describe studies of an index-guided $\sim 4 \times 3 \mu \mathrm{m}^{2}$ vertical cavity surface emitting laser with a cw, room temperature $133 \mu \mathrm{A}$ threshold current and 53\% slope efficiency fabricated using selective wet thermal oxidation to provide optical and electrical confinement. While the device operates strictly single mode, a large number of transverse modes are evident in the subthreshold luminescence observed in both vertical and lateral directions. Despite the lumped nature of the index region, the transverse mode wavelengths agree very well with those predicted for a conventional distributed waveguide structure. Waveguide dimensions and an effective index step of $2 \%$ between the active and cladding region are determined by fitting the modal data. (c) 1995 American Institute of Physics.
\end{abstract}

Recently reported vertical cavity surface emitting lasers (VCSELs) incorporating aluminum oxide layers have featured threshold current reductions to $91 \mu \mathrm{A}^{1}$ and power conversion efficiency increases to $50 \%$. $^{2}$ The low index of the oxide provides optical confinement, evident in the spectra of the latter device, which is critical to realizing smaller devices. In this letter, we investigate waveguiding in a laser of the same structure and fabrication as the high efficiency ones but with smaller area for reduced threshold and single-mode operation. A substantial modal structure, however, is evident in subthreshold luminescence, and it is used to analyze the nature of waveguiding in an aluminum oxide confined surface emitting laser for the first time. The modal dispersion data, which is enhanced in lateral emission spectra, is used to determine the effective dimensions of the waveguide and the size of the effective index step from the active to cladding region.

The laser has been realized by producing a lateral index step and current aperture due to conversion of AlGaAs to an oxide by selective lateral oxidation. ${ }^{3}$ The initial epitaxial structure including $\mathrm{Al}_{0.96} \mathrm{Ga}_{0.04} \mathrm{As} / \mathrm{GaAs}$ distributed Bragg reflectors and a triple InGaAs quantum well in a graded AlGaAs cavity is similar to that described previously, ${ }^{4}$ except that the aluminum mole fraction of the AlGaAs mirror layer closest to the cavity has been increased from $x=0.96$ to $x=0.98$. Devices are formed in mesas created by etching away $\sim 4 \mu \mathrm{m}$ of the surrounding material so as to expose the edge of the cavity layers. A portion of the AlGaAs layers starting from the exposed edge are then converted to an electrically insulating aluminum oxide with a refractive index of $\sim 1.55$ by wet thermal oxidation ${ }^{5}$ at $425^{\circ} \mathrm{C}$ for a few hours. The $x=0.98$ layer oxidizes most rapidly ${ }^{3}$ and the resultant oxide protrudes furthest into the mesa to provide electrical and optical confinement about the active region where this layer has not oxidized. A backside substrate contact and annular contact on the mesa complete the top-emitting laser diode. Figure 1 shows a top view and cutaway orthogonal

${ }^{a)}$ Electronic mail: kllear@sandia.gov view of the laser. The square shape of the oxide aperture (active region) resembles the outline of the mesa from which the oxidation proceeded. Microscopic inspection of the luminescent area indicates an approximately $3 \times 3 \mu \mathrm{m}^{2}$ active area, roughly consistent with the waveguide dimensions extracted below.

The room-temperature, cw light-current characteristic for this laser is presented in Fig. 2. This characteristic was stable with no noticeable changes during experiments over the course of several weeks. It is quite linear in the range shown, reaching a maximum power of $1.5 \mathrm{~mW}$ at $2.5 \mathrm{~mA}$ before rolling over due to heating effects. The threshold current of $133 \mu \mathrm{A}$ is more than an order of magnitude smaller than the minimum threshold observed in lasers made from the same epitaxial material using proton implantation, since such gain guided devices suffer from diffraction losses. ${ }^{6}$ This threshold current has been achieved with relatively high out-
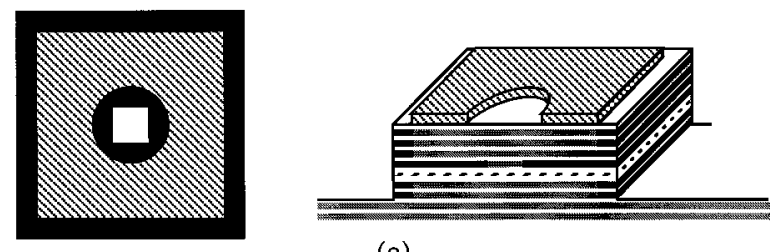

(a)

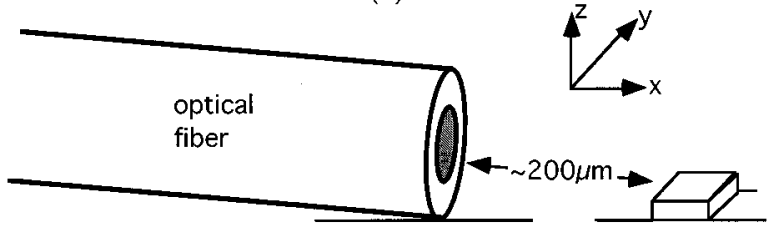

(b)

FIG. 1. (a) Structure of the VCSEL diode shown in top view and cutaway orthogonal view. High aluminum content layers (gray) are converted to oxide (black) including most of the layer directly above the quantum well region (dashed line) except for a small rectangular active region (shown in white in the top view). An annular metal contact (cross hatched) allows for top emission. (b) Arrangement for collecting luminescence from the edge of the laser mesa. The fiber was positioned along the $x$ and $y$ axes in line with the mesa to collect the spectra shown in Figs. 3(a) and 3(b), respectively. 


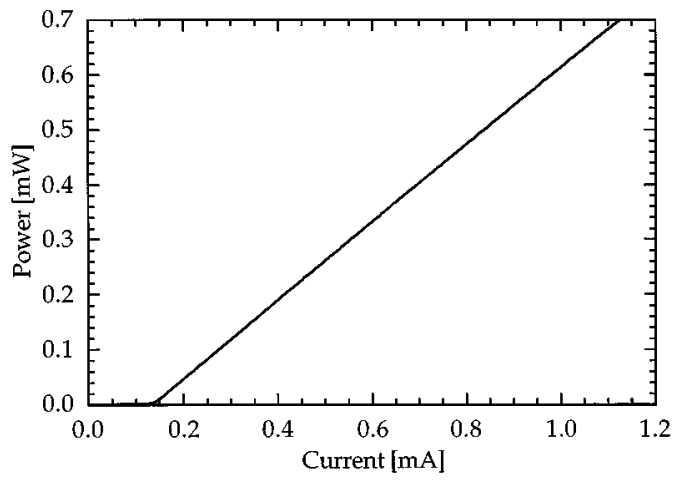

FIG. 2. The light vs current characteristic for a small, index guided, top emitting VCSEL fabricated by selective oxidation.

put coupler transmission so that the differential quantum efficiency just above threshold is 53\%. Higher mirror reflectivity would result in even lower threshold currents. For example, a recently reported, lateral injection, oxide confined VCSEL using a dielectric deposited top mirror exhibited a 91 $\mu \mathrm{A}$ threshold and a slope efficiency of $22 \% .{ }^{1}$ For reference, the lowest reported threshold for an edge-emitting laser is $165 \mu \mathrm{A}$, and this device had a slope efficiency of $0.4 \%{ }^{7}$ The VCSEL reported here lases strictly in the fundamental mode up to and beyond rollover. The moderate effective index step of $2 \%$ in this oxidized device, as compared to the very large semiconductor to air index step in etched devices, promotes the single-mode behavior. In comparison to etched devices, this structure is also more suitable for short wavelength topemitting lasers and offers lower resistance for active region diameters less than about $4 \mu \mathrm{m}$.

While only the fundamental mode reaches lasing threshold, the full modal structure is evident in subthreshold amplified spontaneous emission spectra as shown in Fig. 3. The three spectra corresponding to the emission in the three orthogonal directions were taken at a drive current of $100 \mu \mathrm{A}$. The vertical emission from the surface of the laser was collected using a $0.31 \mathrm{nA}$ microscope objective, while the horizontal emission was collected using a $100 \mu \mathrm{m}$ core optical fiber as indicated in Fig. 1(b). The emission was collected for

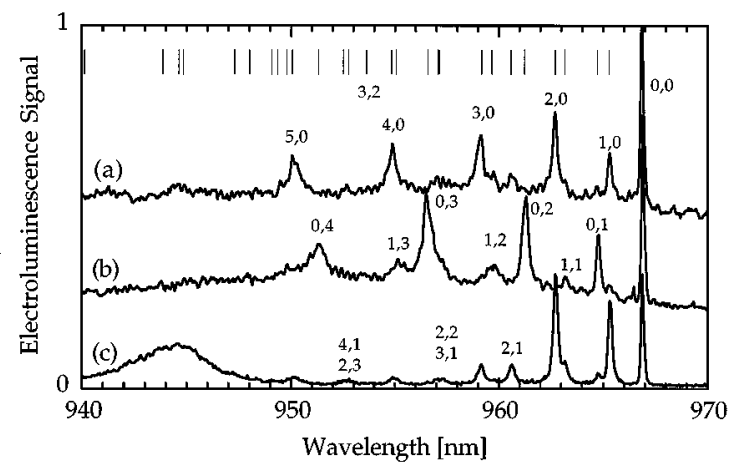

FIG. 3. The subthreshold $(100 \mu \mathrm{A})$ emission spectra for a small VCSEL in the orthogonal horizontal (a) $x$ direction and (b) $y$ direction and in the (c) vertical direction. The computed positions of transverse modes, in excellent agreement with the data, are shown as lines at the top of the figure. The peaks are labeled with the transverse mode order indices determined from the analysis. two orthogonal horizontal directions (along the $x$ and $y$ axes) aligned with the sides of the mesa. The fundamental mode, at the longest wavelength, is observed in the spectra for all three directions. The additional spectral lines are associated with higher order transverse modes, recalling that the onewavelength cavity supports only a single longitudinal mode in the region shown with the next nearest longitudinal mode occurring around $640 \mathrm{~nm}$. The broad feature in the $z$-direction luminescence at $945 \mathrm{~nm}$ corresponds to the Fabry-Perot resonance in the cavity under the oxidized region. The resonance is shifted from that in the unoxidized region, since the change in the index alters the optical cavity length. ${ }^{8}$

Transverse mode filtering in the horizontal direction as well as the small cavity dimensions contribute to horizontal spectra having well-defined transverse mode wavelengths and readily assigned mode indices. The following analysis will verify that the strong, narrow peaks in the horizontal spectra correspond to waveguided modes that are higher order in the direction of emission but are strictly the fundamental mode in the orthogonal lateral direction. We attribute the suppression of higher order orthogonal modes to the spatial filtering of the far field by the limited size of the fiber core which corresponds to selection of wavevectors in the cavity. The detection of higher order orthogonal modes could be enhanced by laterally displacing the fiber position. The small waveguide size leads to modes that are well separated, which improves the relative accuracy of mode wavelengths given a fixed spectrometer resolution. The modal lines in the $x$ direction are more closely spaced than those observed in the $y$ direction indicating a slightly asymmetric (rectangular) waveguide with the $x$ dimension larger than the $y$ dimension.

Based on the following analysis, computed modal positions for the peaks are indicated in Fig. 3. The modes are labeled with the notation $l, m$, where the first index, $l$, is the $x$-component mode order, and the second index, $m$, is the $y$-component mode order. Because of the difference in the phase shift for TM and TE reflections from the waveguide, the two polarizations for a given transverse mode are slightly nondegenerate. The splitting was not resolved in these spectra and is calculated to be small, $1-6 \mathrm{GHz}$, due to the near unity index ratio. In an ideal distributed waveguide structure, the evanescent tails of the confined modes should be strongly attenuated before reaching the outer edges of the mesa. In contrast, the present structure relies on a large index step over a relatively small fraction of the cavity length with no index step for most of the cavity. The low index layer thus acts as a lumped optical element rather than a distributed guide. The strength of the lateral radiation from this structure may indicate that the lumped nature of the waveguiding more effectively scatters or diffracts light out of the waveguide.

A zig-zag ray model ${ }^{9}$ is now used to analyze the observed modal structure and extract waveguide parameters including the lateral dimensions and the index step. Curve fitting of the lateral modal dispersion presented in Fig. 4 allows a determination of the Fabry-Perot wavelength, $k_{z}$, the ratio of the cladding to cavity index, $c=n_{\text {clad }} / n_{\text {cav }}$, and the lateral dimensions, $d_{x}$ and $d_{y}$. The zig-zag ray formulation is 


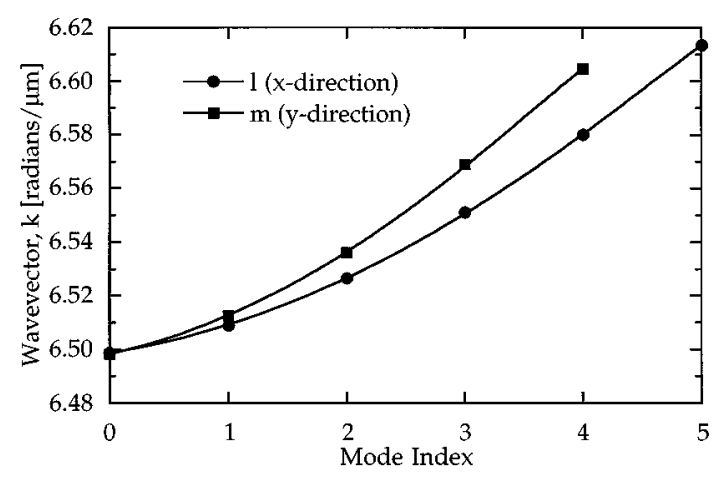

FIG. 4. Modal dispersion for the two horizontal emission spectra. The lines show theoretical fits used to obtain the adjustable parameters of index ratio and waveguide dimension.

used to establish a transcendental equation for the lateral wavevector eigenvalues ${ }^{9}$

$$
\begin{aligned}
& \sqrt{k_{l, m}^{2}-\left(k_{y, m}^{2}+k_{z}^{2}\right)} n_{\mathrm{cav}} d_{x} \\
& \quad-2 \arctan \left(\frac{1}{c^{2 \delta_{\mathrm{TM}}}} \sqrt{\frac{\left(k_{y, m}^{2}+k_{z}^{2}\right)-k_{l, m}^{2} c^{2}}{k_{l, m}^{2}-\left(k_{y, m}^{2}+k_{z}^{2}\right)}}\right)=l \pi,
\end{aligned}
$$

where $\delta_{\mathrm{TM}}=1$ for the TM mode and is zero for the TE mode and the cavity index has been factored out so that the wave-vector values are those external to the cavity where $n=1$. Using the set of data for $k_{l, 0}$ from the $x$-direction spectra, this equation was fit to obtain the three unknown quantities: the effective waveguide width, $n_{\text {cav }} d_{x}=13.09$ $\pm 0.07 \mu \mathrm{m}$, the index ratio, $c=0.9804 \pm 0.0007$, and the magnitude of the orthogonal component of the fundamental wave vector, $\sqrt{k_{y, 0}^{2}+k_{z}^{2}}=6.49514 \pm 0.00009$. The analysis of the $k_{0, m}$ data for the $y$ direction yielded $n_{\text {cav }} d_{y}=10.86 \pm 0.0 \mu \mathrm{m}$, the index ratio, $c=0.9819$ \pm 0.0006 , and the magnitude of the orthogonal component of the fundamental wave vector, $\sqrt{k_{x, 0}^{2}+k_{z}^{2}}=6.49541$ \pm 0.000 19. The fit to the data is excellent as shown in Fig. 4 and quantified with a regression figure of better than $R=0.9999$. The errors in the above parameters are those from the fit. The two sets of data agree well on the index ratio of $c=0.981$. The excellent agreement with the theory for a distributed waveguide is particularly noteworthy since the decreased index layer occupies only $\sim 3 \%$ of the effective cavity length.

The expectation value for the index has been calculated in both the cavity and cladding regions. This effective index value is calculated as the spatial average index weighted by the optical intensity in the cavity, $\langle n\rangle$ $=\left\langle E_{\text {cav }}(z)|n| E_{\text {cav }}(z)\right\rangle /\left\langle E_{\text {cav }}(z) \mid E_{\text {cav }}(z)\right\rangle$, where $E_{\text {cav }}(z)$ is the electric field. The calculated cavity index is $\langle n\rangle_{\text {cav }}$ $=3.26$, so that the physical waveguide cross section is 4.0 $\times 3.3 \mu \mathrm{m}^{2}$. This index also results in an effective index step of $\Delta n=(1-c)\langle n\rangle_{\mathrm{cav}}=0.062$. The effective cladding index, computed using the index profile in the cladding but the electric field distribution of the unoxidized cavity, is $\langle n\rangle_{\text {clad }}=3.16$, giving a calculated index step of 0.10 , in rough agreement with the above value. The uncertainty in the vertical extent of the low index oxide may contribute to the difference in the index step obtained by the two methods. In either case, the index step is much larger than those of $\sim 10^{-3}$ typically employed for lateral confinement in edgeemitting lasers, ${ }^{10}$ but smaller than the huge index step of $>2$ occurring in etched post VCSELs.

The small lateral dimensions of the unoxidized cavity result in a calculated in-plane mode spacing of 30-40 nm at wavelengths around $980 \mathrm{~nm}$. The $2 \%$ effective index step provides only a small reflectivity for these modes, so that lateral cavity $Q$ factor is low. No distinct spectral features are associated with amplified spontaneous emission from these modes, although a broad baseline in the horizontal signals is attributed to broadband lateral spontaneous emission. A factor of 2 to 4 decrease in cavity size would separate in-plane modes sufficiently to place them outside the gain region. This in combination with higher lateral index steps could produce a partial suppression of in-plane spontaneous emission and thus lower the threshold current.

We have demonstrated a small vertical cavity surface emitting laser with optical and electrical confinement provided by a selectively oxidized layer. The laser has a room temperature $133 \mu \mathrm{A}$ threshold current and 53\% slope efficiency. Many transverse modes are clearly seen in the subthreshold luminescence, but the laser operates single mode. The transverse mode wavelengths, taken from threedimensional spectra, have been used to calculate waveguiding parameters including a moderate index ratio of 0.981 for this structure. Yet smaller index steps are desirable for single mode operation in larger diameter devices and can be obtained by positioning the oxidized layer further from the cavity and thus decreasing the field overlap with the oxidized layer. Despite the lumped nature of the index guiding, the transverse modal dispersion agrees extremely well with that of a conventional distributed waveguide.

The authors thank R. Hadley for useful discussions and J. Banas, J. Figiel, K. Geib, and J. Nevers for technical assistance. This work was supported by the United States Department of Energy under Contract No. DE-AC0494AL85000.

${ }^{1}$ D. L. Huffacker, J. Shin, and D. G. Deppe, Electron. Lett. 30, 1946 (1994).

${ }^{2}$ K. L. Lear, K. D. Choquette, R. P. Schneider, Jr., S. P. Kilcoyne, and K. M. Geib, Electron. Lett. 31, 208 (1995).

${ }^{3}$ K. D. Choquette, R. P. Schneider, Jr., K. L. Lear, and K. M. Geib, Electron. Lett. 30, 2043 (1994).

${ }^{4}$ K. L. Lear, R. P. Schneider, Jr., K. D. Choquette, S. P. Kilcoyne, J. J. Figiel, and J. C. Zolper, Photon Technol. Lett. 6, 1053 (1994).

${ }^{5}$ F. A. Kish, S. J. Caracci, N. Holonyak, Jr., J. M. Dallasasse, K. C. Hsieh, S. C. Smith, and R. D. Burnham, Appl. Phys. Lett. 59, 1755 (1991).

${ }^{6}$ K. L. Lear, S. P. Kilcoyne, and S. A. Chalmers, Photon. Technol. Lett. 6, 778 (1994).

${ }^{7}$ T. R. Chen, B. Zhao, L. E. Eng, Y.H. Zhuang, and A. Yariv, in 14th IEEE International Semiconductor Laser Conference (IEEE, Maui, HI, 1994), p. 26.

${ }^{8}$ K. D. Choquette, K. L. Lear, R. P. Schneider, Jr., and K. M. Geib (unpublished).

${ }^{9}$ H. C. Casey, Jr. and M. B. Panish, Heterostructure Lasers (Academic, San Diego, 1978).

${ }^{10}$ G. R. Hadley, Opt. Lett. 14, 308 (1989). 